\title{
DOI: https://doi.org/10.24297/jam.v16i0.8280
}

\author{
New Numerical Methods for Solving Differential Equations \\ Osama. Y. Ababneh \\ Department of Mathematics, Faculty of Science Zarqa University, Zarqa, Jordan \\ osababneh@zu.edu.jo
}

\begin{abstract}
In this paper, we present new numerical methods to solve ordinary differential equations in both linear and nonlinear cases. we apply Daftardar-Gejji technique on theta-method to derive anew family of numerical method. It is shown that the method may be formulated in an equivalent way as a RungeKutta method. The stability of the methods is analyzed.
\end{abstract}

Indexing terms/Keywords : Ordinary Differential Equations, Numerical Method, Iterative Method.

\section{Introduction}

Numerical methods are one of the main techniques used for solving differential equations. For many years, the construction of accurate and stable numerical methods for the solutions of ordinary differential equations (ODEs) with initial value problems has been considered widely and with great new contributions. Recently, the method proposed by Daftardar-Gejji and Jafari (DJM) [1] is powerful technique for solving a wide range of nonlinear equations, see $[2,3,4,5,6,7,8,9,10,11,12,13,14]$. In this paper, we employ the (DJM) to construct a new family of numerical scheme for solving ordinary differential equations and discuss error, stability and convergence of the proposed methods.

\section{Daftardar-Gejji and Jafari Method}

To illustrate the basic concept of the new iterative method, we consider the following general nonlinear system

$u=f+L(u)+N(u)$,

where $f$ is a given function, $L$ and $N$ are linear and nonlinear operators respectively. It is assumed that the [DJM] solution for the Eq. (1) has the form:

$$
u=\sum_{i=0}^{\infty} u_{i}
$$

Since $L$ is linear

$$
L\left(\sum_{i=0}^{\infty} u_{i}\right)=\sum_{i=0}^{\infty} L\left(u_{i}\right) .
$$


The nonlinear operator $\mathrm{N}$ in Eq. (1) is decomposed by [DJM] as bellow:

$$
N\left(\sum_{i=0}^{\infty} u_{i}\right)=N\left(u_{0}\right)+\sum_{i=1}^{\infty}\left\{N\left(\sum_{j=0}^{\infty} u_{j}\right)-N\left(\sum_{j=0}^{i-1} u_{j}\right)\right\}
$$

Using Eqs. (2), (3) and (4) in Eq. (1), we get

$$
\sum_{i=0}^{\infty} u_{i}=f+\sum_{i=0}^{\infty} L\left(u_{i}\right)+\sum_{i=0}^{\infty} G_{i}
$$

The DJM series terms are generated as bellow:

$u_{0}=f, u_{m+1}=L\left(u_{m}\right)+G_{m}, m=0,1,2, \ldots$

The k-term approximate solution is given by

$u=\sum_{i=0}^{k-1} u_{i}$

for suitable integer $\mathrm{k}$.

\section{New Family of Numerical Methods}

Consider the initial value problem

$\frac{d y}{d x}=f(x, y), y^{i}\left(x_{0}\right)=\eta, i=1,2, \ldots, n$,

Where $\quad y:[a, b] \rightarrow R^{n}, \eta \in R^{n}, f:[a, b] \times R^{n} \rightarrow R^{n}$.

Recently, J. Patade et al [15] proposed new method by applying [DJM]on the implicit trapezium method to get a new second order formula and denoted by[NNM]. Now, let us consider the famous family of methods, called by $\theta$-methods which has the following formula

$$
y_{j+1}=y_{j}+h\left[\theta f\left(x_{j}, y_{j}\right)+(1-\theta) f\left(x_{j+1}, y_{j+1}\right)\right], \theta \in[0,1]
$$

where $h=x_{j}-x_{j-1}$ and $x_{j}=x_{0}+j h, j=1,2, \ldots, n$.

We can take different value of $\theta$ in formula (10) to generate many of methods, for example:

$\begin{cases}\theta & =1, \\ \theta & =1 / 2, \quad \text { Explicit } \\ \theta & =0,\end{cases}$

Implicit Euler method. 
We can rewrite formula(10) as the form of(1) by consider

$$
\begin{aligned}
& u=y_{j+1}, \\
& f=y_{j}+h \theta f\left(x_{j}, y_{j}\right), \\
& N(u)=h(1-\theta) f\left(x_{j+1}, y_{j+1}\right)
\end{aligned}
$$

Now, let us apply [DJM] on (10) to get 3-term solution as

$$
\begin{aligned}
& u=u_{0}+u_{1}+u_{2}, \\
& =u_{0}+N\left(u_{0}\right)+N\left(u_{0}+u_{1}\right)-N\left(u_{0}\right), \\
& =u_{0}+N\left(u_{0}+u_{1}\right), \\
& =u_{0}+N\left(u_{0}+N\left(u_{0}\right)\right) .
\end{aligned}
$$

which is

$$
y_{j+1}=y_{j}+h \theta f\left(x_{j}, y_{j}\right)+N\left(y_{j}+h \theta f\left(x_{j}, y_{j}\right)+N\left(y_{j}+h \theta f\left(x_{j}, y_{j}\right)\right) \cdot j=0,1, \ldots\right.
$$

Or

$$
y_{j+1}=y_{j}+h \theta f\left(x_{j}, y_{j}\right)+h(1-\theta) f\left(x_{j+1}, y_{j}+h \theta f\left(x_{j}, y_{j}\right)+h(1-\theta) f\left(x_{j+1}, y_{j}+h \theta f\left(x_{j}, y_{j}\right)\right) .\right.
$$

Therefore, we obtain a new family of $\theta$ method. However, the new family can be formulated in an equivalent way as a RungeKutta method as follow

$$
\begin{aligned}
& k_{1}=f\left(x_{j}, y_{j}\right), \\
& k_{2}=f\left(x_{j+1}, y_{j}+h \theta k_{1}\right), \\
& k_{3}=f\left(x_{j+1}, y_{j}+h \theta k_{1}+h(1-\theta) k_{2}\right),
\end{aligned}
$$

where

$y_{j+1}=y_{j}+h \theta k_{1}+h(1-\theta) k_{3}$.

Now, to obtain some examples for the new family we choose some different values of $\theta$ in (12) as follow:

for $\theta=0$, we get

$k_{1}=f\left(x_{j}, y_{j}\right)$,

$k_{2}=f\left(x_{j+1}, y_{j}\right)$,

$k_{3}=f\left(x_{j+1}, y_{j}+h k_{2}\right)$,

Where

$y_{j+1}=y_{j}+h k_{3}$.

for $\theta=1 / 2$, we get

$k_{1}=f\left(x_{j}, y_{j}\right)$,

$k_{2}=f\left(x_{j+1}, y_{j}+\frac{h}{2} k_{1}\right)$,

$k_{3}=f\left(x_{j+1}, y_{j}+\frac{h}{2} k_{1}+\frac{h}{2} k_{2}\right)$, 
where

which is the method proposed in [15]. for $\theta=3 / 4$, we get

$$
\begin{aligned}
& k_{1}=f\left(x_{j}, y_{j}\right), \\
& k_{2}=f\left(x_{j+1}, y_{j}+\frac{3 h}{4} k_{1}\right), \\
& k_{3}=f\left(x_{j+1}, y_{j}+\frac{3 h}{4} k_{1}+\frac{h}{4} k_{2}\right),
\end{aligned}
$$

where

$$
y_{j+1}=y_{j}+\frac{3 h}{4} k_{1}+\frac{h}{4} k_{3} .
$$

for $\theta=1$, we get

$$
k_{1}=f\left(x_{j}, y_{j}\right) \text {, }
$$

$k_{2}=f\left(x_{j+1}, y_{j}+h k_{1}\right)$,

$k_{3}=f\left(x_{j+1}, y_{j}+h k_{1}\right)$,

Where

$$
y_{j+1}=y_{j}+h \theta k_{1} \text {. }
$$

Theorem. The new family defined by (12) and (14) are of Second order if $\theta=1 / 2$, and first order for any another choice of $\theta$.

Proof: The Taylor series expansion of $y j+1$ may be written as

$$
y\left(x_{j+1}\right)=y_{j}+h f+\frac{1}{2} h^{2} f f_{y}+\frac{1}{6} h^{3}\left(f f_{y}^{2}+f^{2} f_{y y}\right)+O\left(h^{4}\right) .
$$

Notice that for simplicity of the algebra $f$ have been considered as a function of $y$ only, without loss of generality. This will considerably reduce the Taylor series expansions of $k i, i=1,2,3$, in (12) to the following

$$
k_{1}=f,
$$

$$
k_{2}=f+f \theta h f_{y}+\frac{1}{2} \theta^{2} f^{2} h^{2} f_{y y}+\frac{1}{6} \theta^{3} f^{3} h^{3} f_{y y y}+\ldots
$$

$$
k_{3}=f+f_{y}\left(\theta f h+(1-\theta) h\left(f+\theta f h f_{y}+\frac{1}{2} \theta^{2} f^{2} h^{2} f_{y y}+\frac{1}{6} \theta^{3} f^{3} h^{3} f_{y y y}\right)\right)^{2}+\ldots
$$

Traditionally, the equation (19), (20) and (21) would be substituted in (14) to obtain an expression of yj+1. Since the error of the method can be measured using the expression

$T_{j+1}=y\left(x_{j+1}\right)-y_{j+1}$,

therefore, 


$$
T_{j+1}=\left(\theta-\frac{1}{2}\right) f h^{2} f_{y}+\left(\frac{1}{6}-\theta+2 \theta^{2}-\theta^{3}\right) f h^{3} f_{y}^{2}+\left(\frac{\theta}{2}-\frac{1}{3}\right) f^{2} h^{3} f_{y y}+\ldots
$$

Clearly, by choosing $\theta=1 / 2$ we get

$T_{j+1}=\frac{1}{24} f h^{3} f_{y}^{2}-\frac{1}{12} f^{2} h^{3} f_{y y}+O\left(h^{4}\right)$,

which is mean the method is second order, otherwise its first order.

Definition. [16] A scheme is said to be consistent if the difference of the computation formula exactly approximates the differential equation it tends to solve.

Theorem. The new family of modified $\theta$ method is consistent.

Proof: Subtract yj on both sides of (14), and we have:

$$
y_{j+1}-y_{j}=h\left(\theta k_{1}+(1-\theta) k_{3}\right) \text {. }
$$

Dividing all through by $\mathrm{h}$ and taking limit as $\mathrm{h}$ tend to zero on both sides, we have

$$
\lim _{h \rightarrow 0} \frac{y_{j+1}-y_{j}}{h}=\lim _{h \rightarrow 0}\left(\theta k_{1}+(1-\theta) k_{3}\right)=f\left(x_{j}, y_{j}\right) .
$$

Hence, the method is consistent.

\section{The Stability Function for The New Modification Methods}

In order to validate the stability of the method, the equation (12) and (14) are substituted in the simple test equation

$y^{\prime}=\lambda y, \lambda \in C, \operatorname{Re}(\lambda)<0$,

we get

$k_{1}=\lambda y_{j}$,

$k_{2}=\lambda y_{j}(1+\theta \lambda h)$,

$k_{3}=\lambda y_{j}(1+\theta \lambda h+\lambda h(1-\theta)(1+\theta \lambda h))$.

Substituting (27) in (14) and letting $z=h \lambda$, the simplified equation is obtained as follows:

$$
y_{j+1}=y_{j}\left(1+z+z^{2}-\theta z^{2}-\theta z^{3}+2 \theta^{2} z^{3}-\theta^{3} z^{3}\right)
$$

or in more simplified form

$$
\begin{gathered}
y_{j+1}=y_{j} R(z) \\
R(z)=\left(1+z+z^{2}-\theta z^{2}-\theta z^{3}+2 \theta^{2} z^{3}-\theta^{3} z^{3}\right)
\end{gathered}
$$

\section{Conclusion}


In this article, the new family of numerical methods has been successfully obtained. we analyzed the order, consistency and the stability for the new family.

\section{Acknowledgments}

This research is funded by the Deanship of Research in Zarqa University, Jordan.

\section{References}

1. V. Daftardar-Gejji, H. Jafari. An iterative method for solving non linear functional equations. J. Math. Anal. Appl., 2006, 316: 753763.

2. V. Daftardar-Gejji, S. Bhalekar. Solving fractional boundary value problems with Dirichlet boundary conditions. Computers and Mathematics with Applications, 2010, 59(5): 18011809.

3. S. T. Mohyud-Din, A. Yildirim, M. Hosseini. An iterative algorithm for fifth-order boundary value problems.

World Applied Sciences Journal, 2010 8(5): 531535.

4. I. Ullah, H. Khan, M. Rahim. Numerical solutions of higher order nonlinear boundary value problems by new iterative method. Applied Mathematical Sciences, 2013, 7(49): 24292439.

5. I. Ullah, H. Khan, M. T. Rahim. Numerical solutions of fifth and sixth order nonlinear boundary value problems by Daftardar Jafari method. Journal of Computing in Civil Engineering, 2014, 2014.

6. S. Bhalekar, V. Daftardar-Gejji. Solving evolution equations using a new iterative method. Numerical Methods for Partial Differential Equations, 2010, 26(40): 906916.

7. S. Bhalekar, V. Daftardar-Gejji. Solving a system of nonlinear functional equations using revised new iterative method, World Academy of Science, Engineering and Technology, 2012, 6: 127131.

8. S. Bhalekar, V. Daftardar-Gejji. Solving fractional order logistic equation using a new iterative method. International Journal of Differential Equations, 2010 (2012) (Art. ID 975829).

9. S. Bhalekar, V. Daftardar-Gejji. Numeric-analytic solutions of dynamical systems using a new iterative method. Journal of Applied Nonlinear Dynamics, 2012, 1(2): 141158.

10. J. Patade, S. Bhalekar. Approximate analytical solutions of Newell-Whitehead-Segel equation using a new iterative method. World Journal of Modelling and Simulation, 2015, 11(2): 94103.

11. V. Daftardar-Gejji, Y. Sukale, S Bhalekar. A new predictor-corrector method for fractional differential equations. Applied Mathematics and Computation, 2014, 244 (2): 158182.

12. V. Daftardar-Gejji, Y. Sukale, S Bhalekar. Solving fractional delay differential equations: A new approach. Fractional Calculus and Applied Analysis , 2015, 16(2): 400418.

13. H. Jafari, H. Tajadodi, et al. A decomposition method for solving the fractional davey-stewartson equations. International Journal of Applied and Computational Mathematics, 2015: 110. 5

14. A. Hemeda. New iterative method: An application for solving fractional physical differential equations. Abstract and Applied Analysis, Hindawi Publishing Corporation, 2013. 
15. Jayvant Patade and Sachin Bhalekar. A new numerical method based on Daftardar-Gejji and Jafari Technique for solving differential equations. World Journal of Modelling and Simulation Vol. 112015 No. 4, pp. 256-271

16. Ademiluyi, R. A. and Babatola, P. O. 2000. Semi-implicit Runge-Kutta formula for approximation of stiff initial values problem in ODEs. J. Math Sci. Edu. 3:1-2. 